\title{
Spatio-temporal changes in the seismic velocity induced by the 2011 Tohoku-Oki earthquake and slow slip event revealed from seismic interferometry, using ocean bottom seismometer's records
}

\author{
Miyuu Uemura ${ }^{1 *}$ (D) Yoshihiro Ito ${ }^{2}$, Kazuaki Ohta², Ryota Hino ${ }^{3}$ and Masanao Shinohara ${ }^{4}$
}

\begin{abstract}
Seismic interferometry is one of the most effective techniques for detecting temporal variations in seismic velocity caused by large earthquakes. Before the 2011 Tohoku-Oki earthquake $\left(M_{w} 9.0\right)$ near the Japan Trench, a slow slip event (SSE, $\left.M_{w} 7.0\right)$ and low-frequency tremors were observed near the trench. Here, we applied a seismic interferometry technique using ambient noise to data from 17 ocean bottom seismometers (OBSs) installed above the focal region before the main shock. We used our technique to detect temporal variations in seismic velocity caused by the main shock, SSE, and low-frequency tremors. In the region above the large coseismic slip area, we detected a 1-2\% seismic velocity decrease after the main shock. In addition, we observed very small temporal increases in seismic velocity near the SSE fault during the initial SSE stage. Moreover, for most of the OBSs, we observed temporal variations in the autocorrelation functions (ACFs) during the low-frequency tremors. These may have been caused by temporal variations in the ambient noise source distributions, resulting from low-frequency tremors. These results suggest the possibility of detecting low-frequency tremors using ACF monitoring.
\end{abstract}

Keywords: Ambient noise, Autocorrelation function, Seismic interferometry, Slow slip event

\section{Introduction}

Seismic interferometry is one of the most powerful techniques for obtaining Green's functions. While seismic interferometry has a high temporal resolution (equivalent to analyses using an artificial source), it does not require an artificial source. Wegler et al. (2009) reported a decrease in seismic velocity of $0.5 \%$ after the 2004 mid-Niigata earthquake $\left(M_{w} 6.6\right)$ based on seismic interferometry applied to ambient noise. Similarly, other studies have reported decreases in seismic velocity accompanied by large earthquakes in several regions, such as Japan and Sumatra (e.g., Nimiya et al. 2017; Sawazaki et al. 2016; Takagi et al. 2012; $\mathrm{Xu}$ and Song 2009). A wide variety of techniques have been used in many regions, and most studies have reported variations in the seismic velocity structure accompanying large

\footnotetext{
* Correspondence: vemura.miyuu.25c@st.kyoto-u.ac.jp

${ }^{1}$ Kyoto University, Gokasyo, Uji, Kyoto 611-0011, Japan

Full list of author information is available at the end of the article
}

earthquakes. However, only a few studies have reported variations in the underground structure accompanying slow slip events (SSEs) (Rivet et al. 2011, 2014).

A number of studies have reported variations in seismic velocity of between 0.1 and $5 \%$ after a main shock and accompanying SSE. For example, Sawazaki et al. (2016) detected velocity decreases of $3.1 \%$ and $1.4 \% 1$ week after the 2014 northern Nagano Prefecture earthquake $\left(M_{w} 6.2\right)$, and recovery to velocity decreases of $1.9 \%$ and $1.1 \%$ 4 weeks after the main shock, using KiK-net data and autocorrelation functions (ACFs). In contrast, Rivet et al. (2011) reported a velocity decrease of only $0.2 \%$ during the early part of the 2006 Guerrero SSE, and an almost complete velocity decrease recovery during the later part. Seismic velocity decreases immediately after an earthquake, and velocities recover in proportion to the time elapsed. However, during SSEs, changes are observed in 
seismic velocities, which decrease during the early parts of an SSE period and subsequently recover in the later parts.

The 2011 Tohoku-Oki earthquake $\left(M_{w} 9.0\right)$ occurred off the coast of mainland Japan on March 11, 2011, and prior slow earthquakes $\left(M_{w} 7.0\right)$ occurred off the coast of mainland Japan nearer the Japan trench about 1 month prior. Prior to the main shock, a clear preslip was not observed near the main shock's epicenter (Hirose 2011; Hino et al. 2013). An episodic SSE and low-frequency tremors were observed beginning 1 month before the earthquake in the region trenchward of the main shock's epicenter (Ito et al. 2013; Ito et al. 2015). The phase velocity of the Rayleigh wave decreased below $0.5 \%$, accompanying the main shock at Tono, Iwate Prefecture (Takagi et al. 2014). Additionally, accompanying a series of earthquakes (including aftershocks), a seismic velocity decrease of approximately $2 \%$ was detected in southern Fukushima Prefecture (Minato et al. 2012). Moreover, seismic velocity decreases under the sea floor of $1-5 \%$ were reported after the earthquake (Ito and Hino 2013). However, variations in seismic velocity accompanying the preceding SSE have not been reported. Therefore, in this study, we used a seismic interferometry technique using ambient noise to data, and applied it to ocean bottom seismographs (OBSs). We aimed to detect temporal variations in the seismic velocity structure accompanying the SSE.

\section{Methods/Experimental}

\section{Tohoku SSE, analysis period, and data}

From January 29 to March 9, 2011, an SSE and low-frequency tremors were detected offshore from Miyagi Prefecture, Japan (Ito et al. 2013; Ito et al. 2015; Katakami et al. 2018). Low-frequency tremors were observed during three periods:

Sequence 1. January 24-January 29

Sequence 2. February 16-February 20

Sequence 3. March 5-March 9

We used the vertical components of the continuous records of 17 OBSs from November 2010 to April 2011. These OBSs were installed offshore from Miyagi Prefecture before the SSE (Fig. 1), and some were installed above the SSE fault. The OBSs had a sampling rate of $100 \mathrm{~Hz}$ and eigen frequencies of $4.5 \mathrm{~Hz}$. The shallowest OBS was located approximately $300 \mathrm{~m}$ below the sea surface, and the deepest was located approximately $4150 \mathrm{~m}$ below the sea surface (Table 1). The OBSs recorded wave fields with one vertical and two horizontal components, and almost all OBSs recorded data for more than 6 months. However, we used only the data for the vertical components, because the horizontal orientations at the bottom had not been precisely estimated. Moreover, we have not yet detected significant wave propagations between OBSs using Z-Z cross-correlation function (CCF). Therefore, we analyzed only the ACF in the $\mathrm{Z}-\mathrm{Z}$ component.

\section{Data analysis}

Seismic interferometry is one of the most effective techniques for detecting temporal variations in seismic velocities caused by large earthquakes. Claerbout (1968) showed that a reflected wave (generated at an observation station on the earth's surface, reflected off a boundary under the station, and returned to the station) could be obtained by calculating the ACF from the waveform observed at the station. This technique is only effective if the structure around the site is a horizontal stratified structure. In the field of helioseismology, Duvall et al. (1993) proved that the Green's function between two stations can be obtained by calculating the CCF between the stations. In seismic interferometry, this technique is applied to the field of seismology. Since Campillo and Paul (2003) reported that a CCF constructed from teleseismic coda waves corresponded to the direct wave between two stations in the scattering field, many studies using seismic interferometry have been published (e.g., Hillers and Campillo 2016; Poli et al. 2012).

In seismic interferometry, a Green's function is created by assuming that one station is the hypocenter and another station is an observation station. This is achieved by calculating the CCF between the waveforms of the wave field at each station (e.g., Wapenaar et al. 2010). Assuming a noise source that is uniformly distributed around the stations (both temporally and spatially), the CCF $(r(\tau))$ between the two stations is calculated using the following formula:

$$
r(\tau)=\frac{\int_{-\infty}^{\infty} s(t) u(t+\tau) d t}{\sqrt{\int_{-\infty}^{\infty} s(t)^{2} d t \int_{-\infty}^{\infty} u(t)^{2} d t}}
$$

where $s(t)$ and $u(t)$ are the observation waveforms at the two stations. If seismic interferometry is applied to only one observation station (i.e., the ACFs of the station are calculated by replacing $u(t)$ with $s(t)$ in Eq. (1)), then a waveform can be derived by stacking the ACFs. The waveform is generated at the station, reflected by a boundary surface under the station, and returned to the station.

We removed the waveforms of ordinary earthquakes from the analysis, because apparent variations in ambient noise can affect the ACF and CCF values. These ambient noise variations are caused by temporal changes in background seismicity, such as variations in the hypocenter distribution of ambient noise. To exclude the effects of the spatio-temporal variations caused by the temporal changes in background seismicity, we applied weight functions based on a seismic coda wave shape for ordinary earthquakes (Katakami et al. 2017). Generally, regardless of the distance from the hypocenter, after the elapsed time (which is twice the travel time of an $\mathrm{S}$ wave), the envelope 


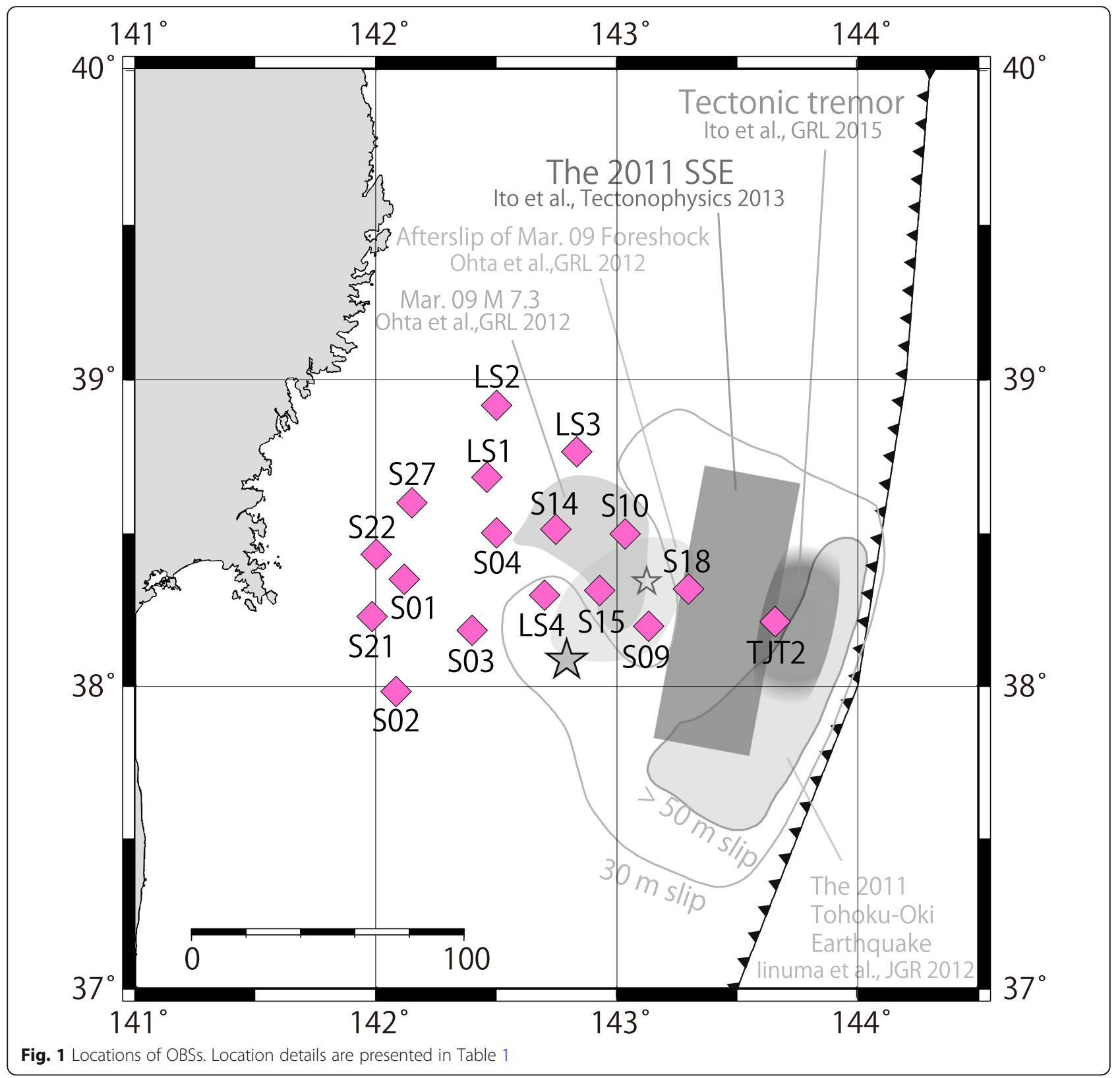

form $s(t)$ of a seismic coda wave satisfies the following formula:

$$
s(t) \propto \exp [-\pi f t / Q]
$$

where $t$ is the time elapsed since the earthquake, and $f$ and $Q$ are the frequency and attenuation factors within the region, respectively (Rautian and Khalturin 1978). Here, we use a simplified function from Eq. (2) and assume an attenuation factor of 50 , which was determined experimentally:

$$
s_{S}(t)=\exp (-t / 50)
$$

In addition, we calculated the envelope of the observed seismogram $s_{O}(t)$ from one vertical and two horizontal components of the continuous record for 1 day:

$$
s_{O}(t)=\sqrt{\sum_{i=1}^{3} s_{i}^{2} /}
$$

Furthermore, we calculated the coefficient $c(t)$ of the cross-correlation between the observed envelope $s_{S}(t)$ and the calculated envelope $s_{O}(\tau)$ using a moving time 
Table 1 Location and recording period of each OBS. We measured the locations of LS1 and S03 when they were collected, and the locations of other stations when they were set up

\begin{tabular}{|c|c|c|c|c|}
\hline \multirow[t]{2}{*}{ Station name } & \multicolumn{3}{|c|}{ Location of OBSs } & \multirow[t]{2}{*}{ Record period } \\
\hline & Longitude ( $\left.{ }^{\circ} \mathrm{E}\right)$ & Latitude $\left({ }^{\circ} \mathrm{N}\right)$ & Depth from sea surface $(\mathrm{m})$ & \\
\hline LS1 (Additional file 1, Additional file 2) & 142.46059 & 38.68408 & 1112 & November 5, 2010-April 13, 2011 \\
\hline LS2 (Additional file 3, Additional file 4) & 142.49997 & 38.91679 & 1194 & November 5, 2010-October 6, 2011 \\
\hline LS3 (Additional file 5, Additional file 6) & 142.83307 & 38.76621 & 1403 & November 5, 2010-October 6, 2011 \\
\hline LS4 (Additional file 7, Additional file 8) & 142.69956 & 38.29971 & 1409 & November 5, 2010-October 6, 2011 \\
\hline S01 (Additional file 9, Additional file 10) & 142.11688 & 38.35023 & 524 & November 5, 2010-May 21, 2011 \\
\hline S02 (Additional file 11, Additional file 12) & 142.08274 & 37.98356 & 538 & November 5, 2010-May 21, 2011 \\
\hline S03 (Additional file 13, Additional file 14) & 142.39969 & 38.18343 & 1052 & November 5, 2010-April 13, 2011 \\
\hline S04 (Additional file 15, Additional file 16) & 142.50041 & 38.50208 & 1100 & October 1, 2010-July 12, 2011 \\
\hline S09 (Figure 2, Figure 3) & 143.13214 & 38.19742 & 2041 & October 1, 2010-July 12, 2011 \\
\hline S10 (Figure 4, Additional file 17) & 143.03415 & 38.49841 & 1981 & July 1, 2010-March 18, 2011 \\
\hline S14 (Additional file 18, Additional file 19) & 142.7457 & 38.51379 & 1459 & July 1, 2010-May 22, 2011 \\
\hline S15 (Additional file 20, Additional file 21) & 142.9276 & 38.31377 & 1454 & July 1, 2010-May 22, 2011 \\
\hline S18 (Additional file 22, Additional file 23) & 143.29608 & 38.31918 & 2770 & July 1, 2010-March 18, 2011 \\
\hline S21 (Figure 3, Additional file 24) & 142.00192 & 38.4319 & 358 & November 5, 2010-May 21, 2011 \\
\hline S22 (Additional file 25, Additional file 26) & 141.98375 & 38.22922 & 299 & November 5, 2010-May 21, 2011 \\
\hline S27 (Additional file 27, Additional file 28) & 142.15009 & 38.60032 & 545 & November 5, 2010-May 21, 2011 \\
\hline TJT2 (Additional file 29, Additional file 30) & 143.65558 & 38.21309 & 4147 & November 19, 2010-May 23, 2011 \\
\hline
\end{tabular}

window with a length of $300 \mathrm{~s}$. We applied the weight $w(t)$ to each time window for the ACF calculations, according to the cross-correlation coefficient as follows:

$$
w(t)=\left\{\begin{array}{l}
0(c(t)>0.5) \\
1(c(t) \leq 0.5)
\end{array}\right.
$$

Generally, the coefficient $c(t)$ represents the correlation between the observed and calculated envelopes only after an elapsed time that is twice the travel time of the $\mathrm{S}$ wave. In addition, we also applied a weight of 0 before $300 \mathrm{~s}$ if the coefficient exceeded 0.5, because the $600 \mathrm{~s}$ time window included seismic waves radiating from the ordinary earthquake.

In this study, we investigate spatio-temporal variations of three functions: ACFs of ambient noise, cross-correlation coefficients of the CCFs between a 15-day ACF and a reference ACF, and phase shift based on the CCFs. These values are calculated as shown below.

First, all observation data were corrected for the instrument response before applying the band-pass filter. Then, we applied a band-pass filter at $0.25-2.0 \mathrm{~Hz}$ and a one-bit technique to the observed continuous data. These filters were applied to prevent the continuous data from being affected by (1) unexpected signals (such as micro-earthquakes) that could not be removed with the aforementioned weight function, or (2) mechanical or biological noise at the site.
Next, after applying the band-pass filter and one-bit technique at intervals of $0.1 \mathrm{~s}$, we calculated $120 \mathrm{~s} \mathrm{ACFs}$ using $5 \mathrm{~s}$ moving time windows. The coefficient for the time including earthquakes equaled 0 after multiplying the weight $w(t)$ of the time window, and the earthquake effects were removed. The 15-day ACF was defined as the ensemble average of the $120 \mathrm{~s}$ ACFs calculated over 15 days. The reference ACF was defined as the ensemble average of $120 \mathrm{~s}$ ACFs calculated over a month from November 19 to December 19, the period before the occurrence of SSE and low-frequency tremors.

In addition, we calculated the CCF using a $15 \mathrm{~s}$ moving time window between the 15-day ACF and reference ACF with a lag time of $-2 \mathrm{~s}$ to $2 \mathrm{~s}$, at intervals of $0.1 \mathrm{~s}$, and estimated the variation in seismic velocity based on the lag time at the maximum CCF. Finally, we objectively evaluated the temporal variations in the 15-day ACFs based on the variations in the cross-correlation coefficient at zero lag time. The shape changes of the 15-day ACFs cause the coefficients to decrease. When a velocity change occurs, the phase of the 15-day ACF delays or progresses, and the shape changes. Even when a velocity change does not occur, it is possible for the shape to change, and a variation in the hypocenter distribution of the ambient noise may explain this shape changes. Therefore, we used the coefficient at zero lag time to detect the temporal variations in the 15-day ACFs caused by, not only velocity change, but also temporal variation of the ambient noise. 


\section{Results}

\section{Temporal variations in ACF}

We detected several temporal variations accompanying the 2011 Tohoku-Oki earthquake, SSE, and low-frequency tremors. The cross-correlation coefficient at zero lag time between the 15-day and reference ACFs during later lapse times decreased after the main shock (Fig. 2b). This decrease was clearer at trenchward stations (e.g., S09 (Fig. 2b) and S10 (Fig. 4b)) than landward stations (e.g., S21 (Fig. $3 \mathrm{~b})$ ), and continued until the end of the observation period without the full recovery of the pre-main shock coefficient value. At nine stations (TJT2 (Additional file 29(b)), S15 (Additional file 20(b)), S14 (Additional file 18(b)), S09, S04 (Additional file 15(b)), LS4 (Additional file 7(b)), LS3 (Additional file 5(b)), LS2 (Additional file 3(b)), and LS1 (Additional file 1(b))), we detected decreases in the coefficient between the 15-day and reference ACFs after the main shock. We excluded landward stations, such as S21 (Fig. 3b), and stations collected 1 week after the main shock, such as S10 (Fig. 4b).

The cross-correlation coefficient around a lapse time of $10 \mathrm{~s}$ decreased after sequence 1 (Fig. 2b). A decrease in the coefficient was detected at most stations installed east of $142.5^{\circ} \mathrm{E}$ and appeared before sequence 1 at $60 \%$ of these stations, such as S10 (Fig. 4b). At all stations, the decreases recovered after 10 days. Similar coefficient variations were detected during sequences 2 and 3, but the decreases were less than that during sequence 1. During SSE, some sustained variations in cross-correlation coefficients were not detected.

\section{Temporal variations in seismic velocity based on} variations in the 15-day ACF

We measured phase delays and progressions in 15-day ACFs after the main shock. If the phase of the 15-day ACF was delayed from the phase of the reference ACF, we called this "phase delay," and if the phase of the 15-day ACF proceeded from the phase of the reference ACF, we called this "phase progression." At station S09, the phase delay during the later lapse time was larger than it was during the early lapse time (Fig. 2c). Velocity changes $\frac{d v}{v}$ around the site were calculated from phase changes using the following formula:

$$
\frac{d v}{v}=-\frac{d T}{T}
$$

where $T$ is the lapse time and $d T$ is the phase progression at a lapse time of $T \mathrm{~s}$ (Snieder et al. 2002). For example, the phase delay between a lapse time of $40 \mathrm{~s}$ and $50 \mathrm{~s}$ was approximately equivalent to a $2 \%$ velocity decrease (Fig. 5). This decrease was detected in multiple lapse times. While the velocity recovered slightly over time after the main shock, it did not recover completely by the end of the study period for all lapse times. Similar velocity decreases were detected at five stations near the trench (TJT2 (Additional file 30), S15 (Additional file 21), S04 (Additional file 16), LS1 (Additional file 2), and LS4 (Additional file 8)). These post-main-shock decreases were approximately $1-2 \%$ and did not recover completely. An OBS was installed closer to the epicenter of the main shock, and greater velocity decreases were detected.

In addition, we also calculated the velocity changes accompanying the SSE and low-frequency tremors from the phase changes. However, we did not detect obvious changes. Although velocities changed by a few tenths of a percent during the SSE at several stations, such as S09 and S15, the changes were equivalent to those in a normal period when no SSE, no low-frequency tremors, and no large earthquakes occurred (Fig. 5). At all stations, the velocity changes accompanying the SSE and low-frequency tremors were smaller than the velocity fluctuations in the normal period.

\section{Regional characteristics of temporal variations in 15-day ACF}

Next, we assessed decreases in the cross-correlation coefficient between the 15-day ACFs and the reference $\mathrm{ACF}$ at zero lag time around a lapse time of $10 \mathrm{~s}$ during the occurrence of low-frequency tremors. We calculated the average coefficients for the seven periods, shown in Table 2, using a $15 \mathrm{~s}$ moving time window from a lapse time of $2.5 \mathrm{~s}$ to $17.5 \mathrm{~s}$ (Fig. 6).

At the six landward stations (S27, S22, S21, S02, S01, and LS1), the variations for each period were extremely small. This indicates that the cross-correlation coefficients at the six stations were stable in all periods (hereafter region L). However, at the other 11 stations, clear variations were observed for each period. For example, the variation from period II to period III (Fig. 6b) was equivalent to the variation induced by a low-frequency tremor occurrence. A low-frequency tremor did not occur in period II but did occur in period III. At the 11 stations, the variations basically decreased when low-frequency tremors occurred and increased (recovered) after low-frequency occurrences.

The 11 stations were classified into 2 groups. In one group, the coefficient decreased primarily in period II. The eight stations (S15, S14, S10, S04, S03, LS4, LS3, and LS2) located at the center of the OBS array (hereafter Region $C$ ) belong to this first group. In another group, the coefficient decreased primarily in period III. The three stations (TJT2, S18, and S09) located near the trench (hereafter Region $\mathrm{T}$ ) belong to this second group. Regions $\mathrm{C}$ and $\mathrm{T}$ correspond roughly to the slip area of the largest foreshock and the SSE occurrence area mentioned in Ito et al. (2013), respectively. 

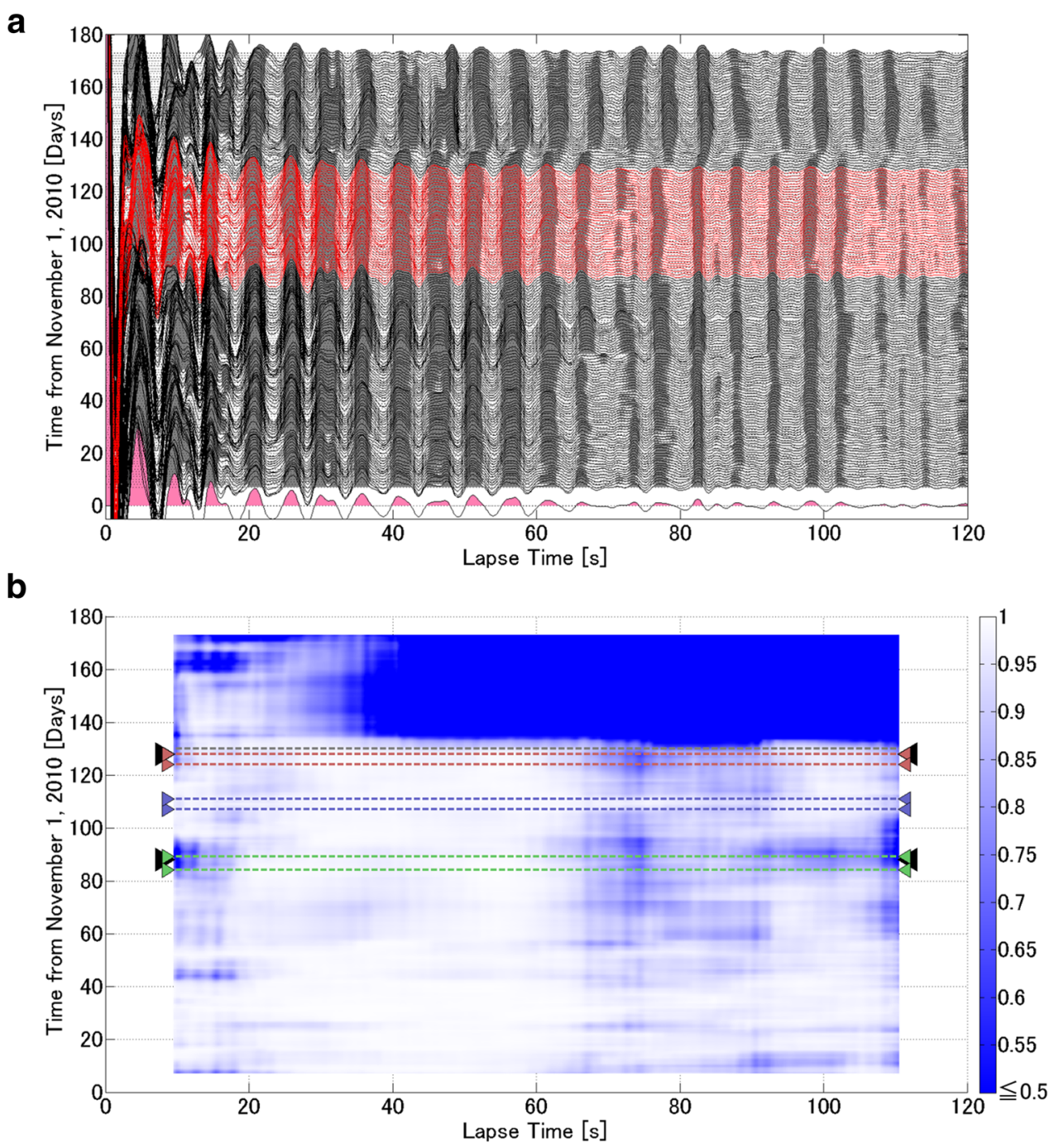

c

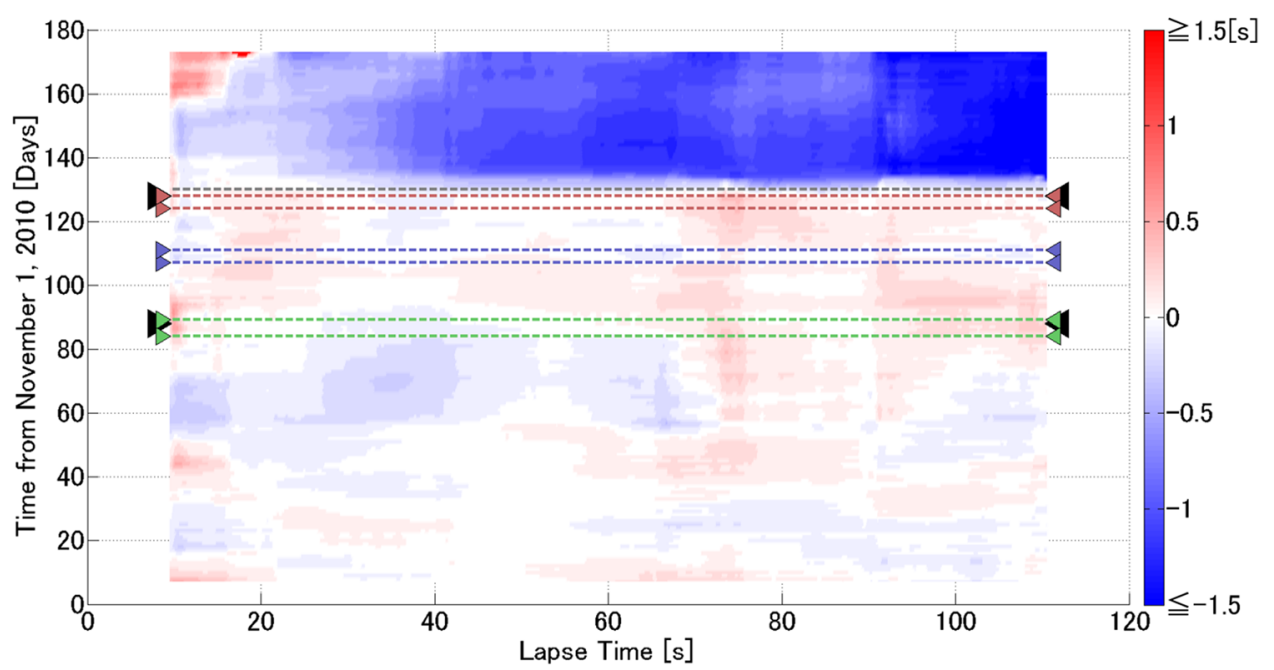

Fig. 2 (See legend on next page.) 
(See figure on previous page.)

Fig. 2 Temporal variations in 15-day ACFs at station S09. a 15-day ACFs calculated from OBS data over 15 continuous days, including the 7 days before and after the given date. The ACFs plotted in red are in the SSE period, and the bottom ACF is the reference ACF, calculated for the month from November 19 to December 19. b The cross-correlation coefficient between the 15-day ACFs and the reference ACF, using a 15-s time window and zero lag time. The period between the black triangles corresponds to the SSE period, and the periods between the red, blue, and green triangles correspond to low-frequency tremor periods in sequences 1,2, and 3. The magenta broken line represents the main shock. c Phase variation in the 15day ACFs relative to the reference ACF, using a 15-s time window. Warm colors represent phase progressions, and cold colors represent phase delays. The four pairs of triangles and the magenta broken line correspond to each period and the main shock described in (b)

\section{Discussion}

Temporal variations in seismic velocity and the 15-day ACF

The observed phase delays after the main shock were larger during the later lapse times for the 15-day ACFs than during the earlier lapse times (Fig. 2c). When velocity structures change equally, the travel time increases monotonically for the wave transmitted in a region in proportion with the propagation distance, resulting in an increase in the phase delay during the latter part of the 15-day ACF. In addition, the phase in the latter part of the 15-day ACF carries information regarding waves that have traveled longer distances because of reflection and refraction around the observation station. Moreover, it is possible for the velocity to change equally around a site after the main shock. At S09, the equal velocity decreased after the main shocks were detected for the lapse times of 30$40 \mathrm{~s}, 40-50 \mathrm{~s}, 50-60 \mathrm{~s}$, and 60-70 s (Fig. 5). This suggests that a wave reflected many times under S09 reaches this observation station after $60 \mathrm{~s}$. In contrast, the phase delay at TJT2 appeared only for part of the lapse time (Additional file 1), suggesting that the velocity structure did not change equally, but rather in part of the region.

At S09, although phase changes appeared for only a short section around a lapse time of $10 \mathrm{~s}$ during sequences 1,2 , and 3 (Fig. 2c), we did not detect a velocity change corresponding to the phase changes (Fig. 5). If the phase changes are caused by an increase in the seismic velocity in part of the region, a phase (or velocity) change several times larger than a phase change at a lapse time of $10 \mathrm{~s}$ should appear when the wave transmitted along the same path several times returns to the station. However, such a phase change was not observed. Therefore, the phase changes were not caused by variations in the velocity structure, and it is possible that variations in the hypocenter distribution of ambient noise induced by the low-frequency tremors cause a phase change similar to that caused by a structural change. The phase progression during sequence 1 continued longer after the end of the low-frequency tremor activity and was larger than those during sequences 2 and 3. This phase progression probably included the dummy structural variation accompanying the beginning of the SSE, in addition to the dummy structural variation caused by the variation in the hypocenter distribution accompanying the low-frequency tremors.
When the density of a crack near a hypocenter increases because of the formation of cracks before an earthquake, seismic velocity is expected to decrease around the hypocenter. Several studies using rocks have reported a decrease in the elastic wave velocity accompanying an increase in crack density (e.g., Nishizawa and Kanagawa 2005). According to Lockner et al. (1991), a decrease in elastic wave velocity and decrease in amplitude were detected before a rock sample broke. This was accompanied by the creation of micro-cracks formed by acoustic emissions. Therefore, we speculate that the observed phase delays and decreases in cross-correlation coefficients may have been caused by the creation of micro-cracks. We detected decreases in velocity before the main shock, and noted that these velocity decreases began earlier at the observation station nearer the hypocenter of the main shock. However, we detected no velocity changes before the SSE around the SSE region. This suggests the possibility that the crack density varied little before the SSE, although the density varied enough to change the 15-day ACF before the main shock. Overall, the results suggest that the mechanism of structural change in seismic velocity accompanying an SSE detected by seismic interferometry may differ from that associated with an earthquake.

\section{Spatio-temporal variations in cross-correlation coefficients at each station}

We classified the temporal variations in the cross-correlation coefficients around a lapse time of $10 \mathrm{~s}$ at all stations (Fig. 6) into three groups: region T, region $\mathrm{C}$, and region $\mathrm{L}$. These three regions corresponded to three slip areas: region $\mathrm{L}$ corresponded to the area that slipped only during the main shock (Iinuma et al. 2012), region $\mathrm{C}$ corresponded to the slip area of the largest foreshock (Ohta et al. 2012), and region T corresponded to the SSE slip area (Ito et al. 2013). In "Temporal variations in seismic velocity and the 15-day ACF" section, we indicated the possibility that the phase changes around a lapse time of $10 \mathrm{~s}$ were caused by the occurrence of low-frequency tremors. The cross-correlation coefficient between the 15-day ACF and the reference ACF at zero lag time decreases if the phase of the 15-day ACF changes. Although the coefficients decreased during sequences 1,2 , and 3 , the time when the decrease of sequence 1 began was different at each 


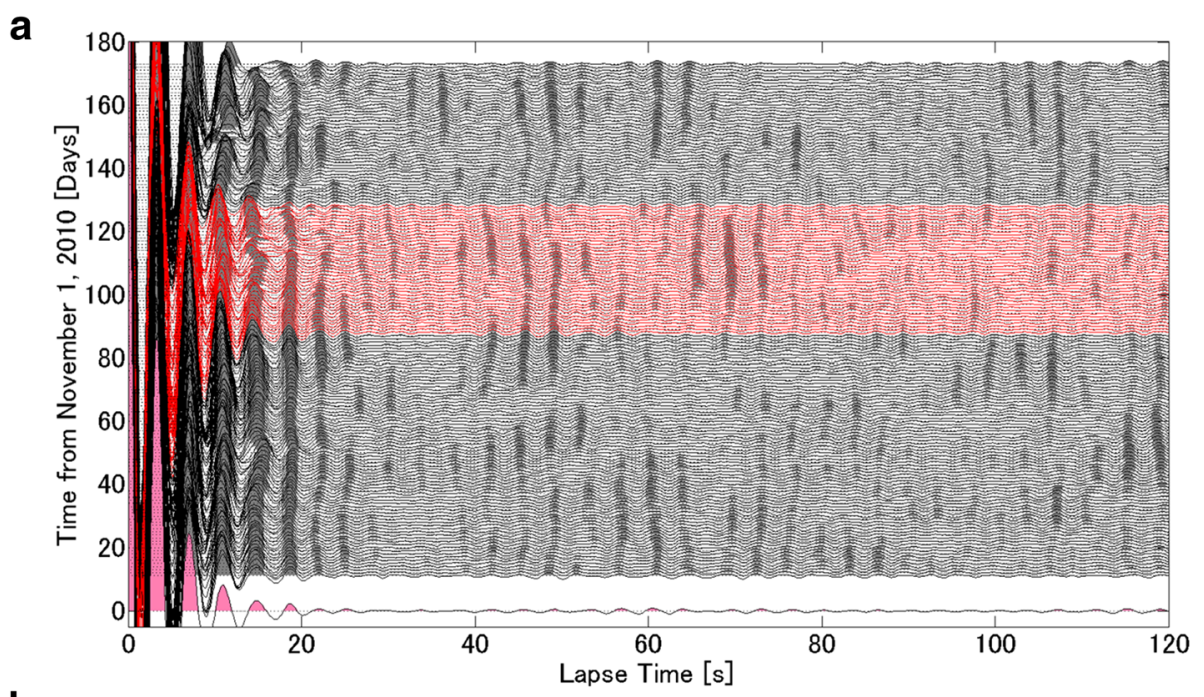

b

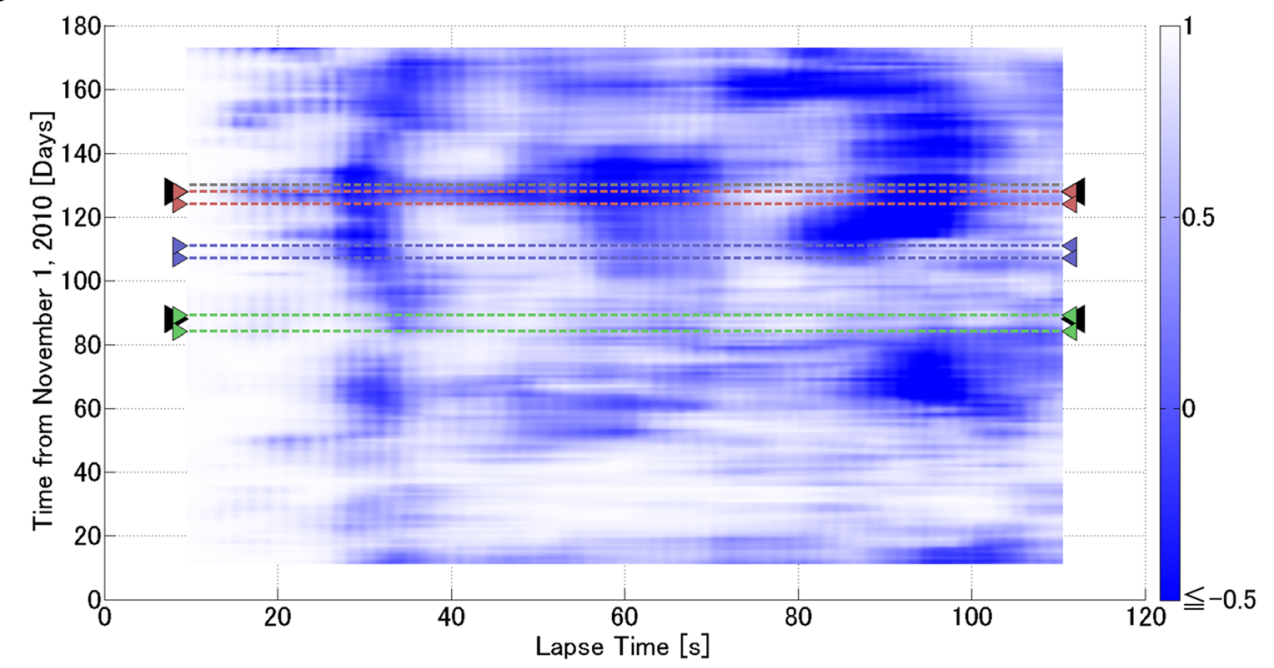

C

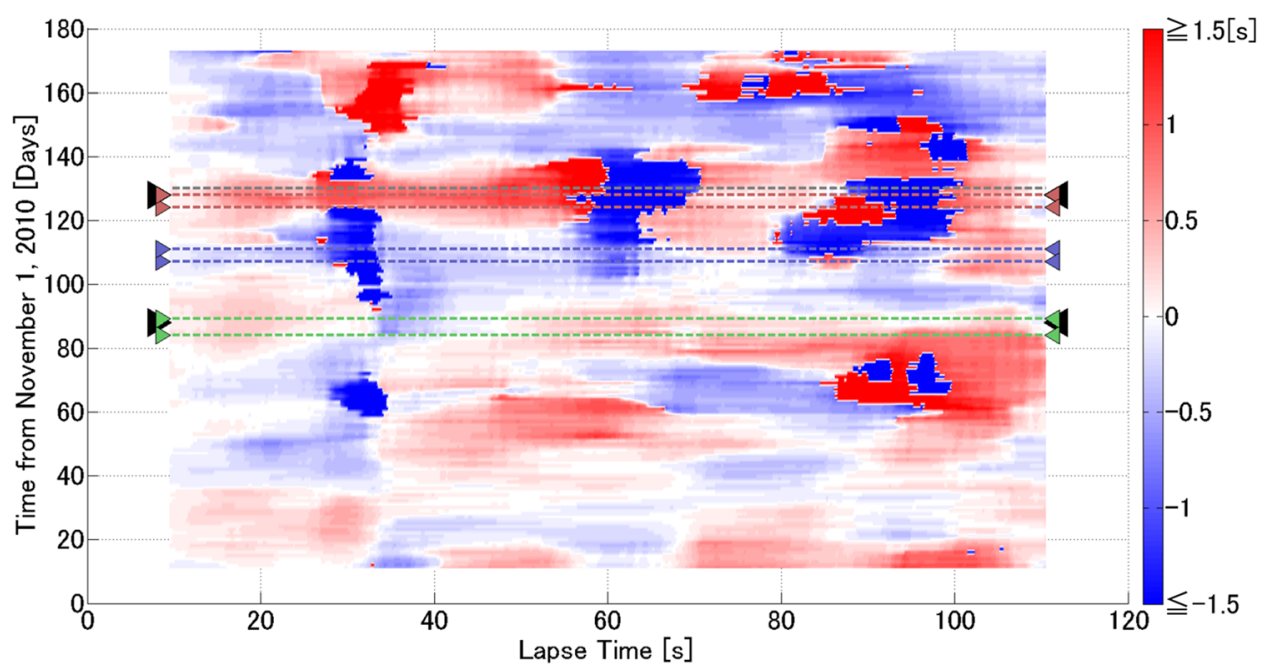

Fig. 3 (See legend on next page.) 
(See figure on previous page.)

Fig. 3 Temporal variations in 15-day ACFs at station S21. a 15-day ACFs calculated from OBS data over 15 continuous days, including the 7 days before and after the given date. The ACFs plotted in red are in the SSE period, and the bottom ACF is the reference ACF, calculated for the month from November 19 to December 19. b The cross-correlation coefficient between the 15-day ACFs and the reference ACF, using a 15-s time window and zero lag time. The period between the black triangles corresponds to the SSE period, and the periods between the red, blue, and green triangles correspond to low-frequency tremor periods in sequences 1,2 , and 3 . The magenta broken line represents the main shock. c Phase variation in the 15-day ACFs relative to the reference ACF, using a 15-s time window. Warm colors represent phase progressions, and cold colors represent phase delays. The four pairs of triangles and the magenta broken line correspond to each period and the main shock described in (b)

observation station. In region $\mathrm{C}$, where coefficient decreases started from period II before sequence 1 , it is possible that low-frequency tremors occurred during period II, and that the 15-day ACF changed because of variations in the hypocenter distribution. In agreement, Katakami et al. (2018) reported that micro-low-frequency tremors occurred near region $\mathrm{C}$ in the same period. Therefore, the coefficient decreases during period II were caused by the occurrence of a low-frequency tremor.

Meanwhile, the widths of the lapse time section, where the coefficients decreased substantially, were different in during period II and during low-frequency tremor periods (III, V, and VII). The coefficients during period II at the stations installed in region $C$ decreased substantially in a wide section between lapse times of $10 \mathrm{~s}$ and $20 \mathrm{~s}$ (Fig. 4b). However, the coefficients during periods V and VII at the stations installed in region $C$ decreased substantially in narrow sections around a lapse time of $10 \mathrm{~s}$. The coefficients during period III at stations installed in region $\mathrm{T}$ also decreased largely in a narrow range, around a lapse time of $10 \mathrm{~s}$ (Fig. 2b). The width of the lapse time section during period II was different from the widths of the lapse-time section during periods V and VII at the same station installed in region $C$. In addition, although the stations were different, the width of the lapse time section during period II at the station installed in region $C$ was different from the widths of the lapse time section during period III at stations installed in region T. This suggests that the decrease during period II was unique in terms of the width of the lapse time section where the coefficients decreased substantially compared to the coefficient decreases for the section widths during periods III, V, and VII. Therefore, it is possible that the decrease during period II occurred not only because of low-frequency tremors, as detected by Katakami et al. (2018), but also because of an additional event, for example, the preparation process of the 2011 Tohoku-Oki earthquake.

\section{Estimated velocity reductions during 2011 Tohoku-Oki earthquake and SSE}

The decrease in velocity under an observation station represents one interpretation of the cause of a phase delay. When a wave reflects off a surface and returns to the station, a phase delay is observed if the seismic wave velocity decreases along the propagation path. The delay can also be explained by an increase in the distance between an observation station and a reflection surface, because the propagation distance of the reflected wave is determined by the distance between an observation station and a reflection surface. Assuming that the distance to the reflection surface did not change, the observed phase delays after the main shock were equivalent to a $2 \%$ or lower seismic velocity decrease at six stations (TJT2, S15, S09, S04, LS4, and LS1) near the trench. Ito and Hino (2013) studied the sea floor in the same region as the present study, and detected decreases in seismic velocity of $1-5 \%$ after the main shock in the overall analysis region. Moreover, a decrease in seismic velocity of $2 \%$ in the southern Fukushima Prefecture (Minato et al. 2012) and $0.1-0.5 \%$ in the Iwate Prefecture (Takagi et al. 2012) were reported after the main shock using land data. In the present study, the observed seismic velocity decreases were lower than those of Ito and Hino (2013), but roughly equivalent to those of other regions that shook during the main shock.

In the SSE period, we detected a perturbation in seismic velocity of a few tenths of a percent around the SSE area. At TJT2, S18 (Additional file 23), and S09, which were installed near the SSE region, the velocity changes calculated from the phase changes at lapse times of 20-30 s, 30-40 s, and 40-50 s increased slightly during the initial SSE stage, and did not change after that stage (e.g., Fig. 5). Two studies of the 2006 SSE and 2009 SSE at Guerrero, Mexico, detected decreases and recoveries in seismic velocity during the SSEs using data from stations landward of the SSE slip regions (Rivet et al. 2011, 2014). The researchers proposed that the velocity decreased by $0.8 \%$ in the former part of the SSE period and recovered almost completely in the latter part. In this study, increases in velocity were observed in the former part of the SSE period using data from both landward and trenchward stations. However, after that, we did not observe an additional decrease (recovery) during the observation period. We observed a difference between the two Guerrero SSEs and the Tohoku-Oki SSE with respect to whether or not any large earthquakes occurred at the plate boundary immediately after the SSEs. The Guerrero SSEs concluded without any large earthquakes, but the Tohoku-Oki SSE concluded with large earthquakes with foreshocks, a 
a

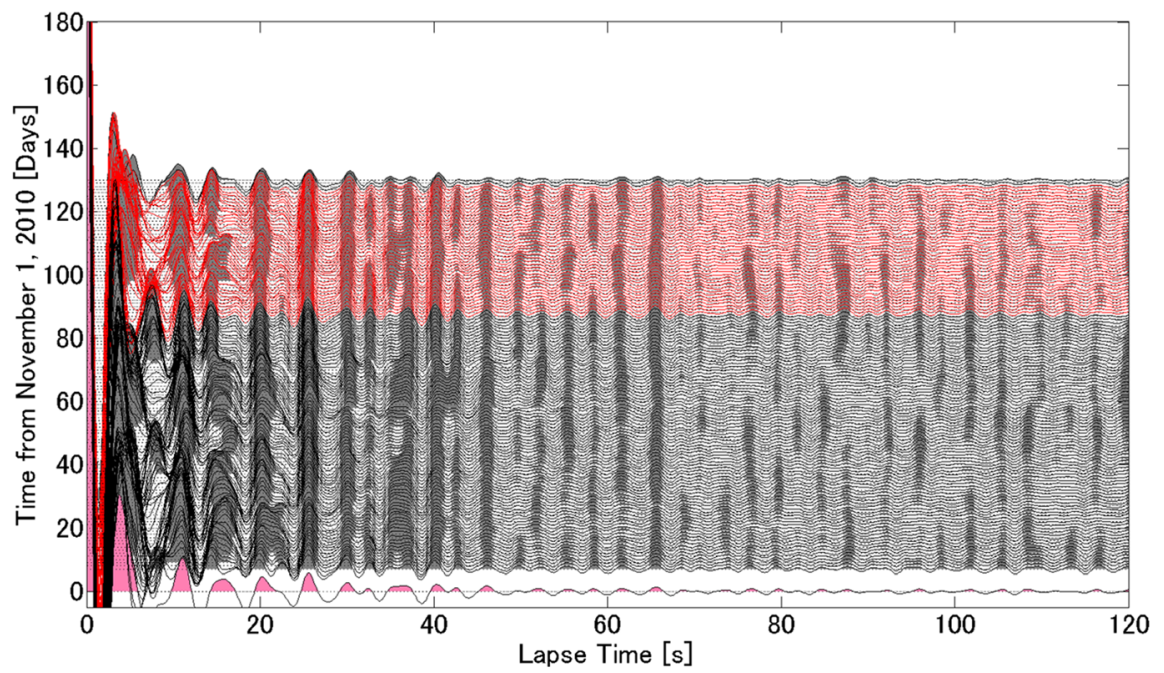

b

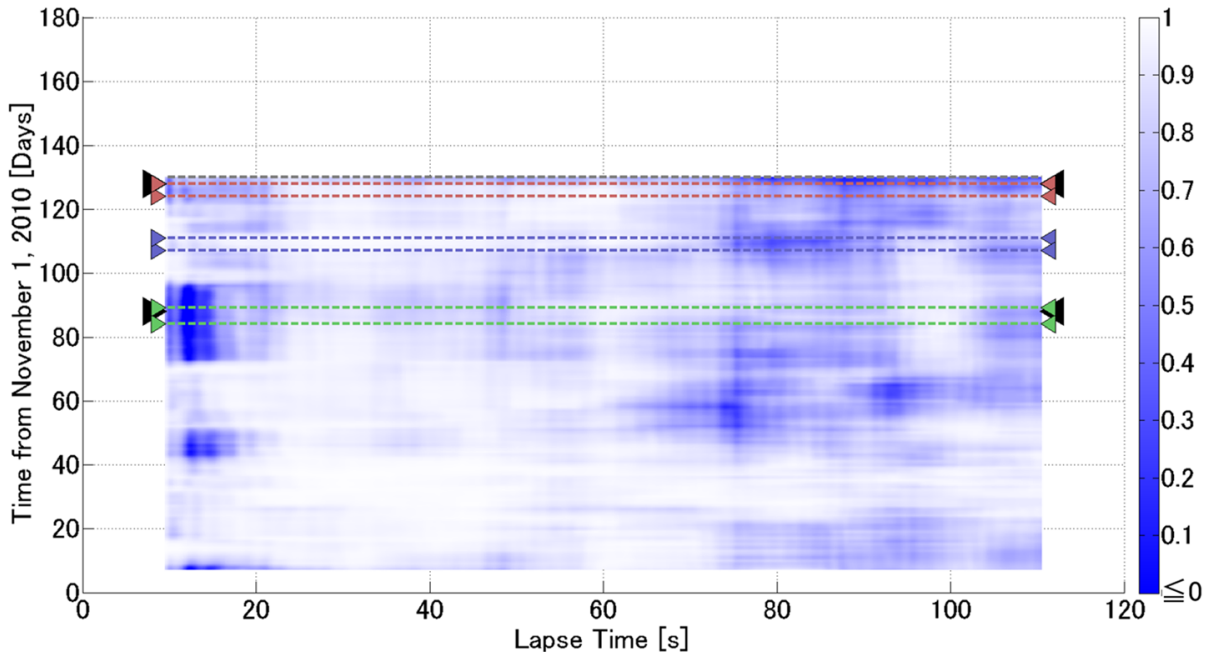

C

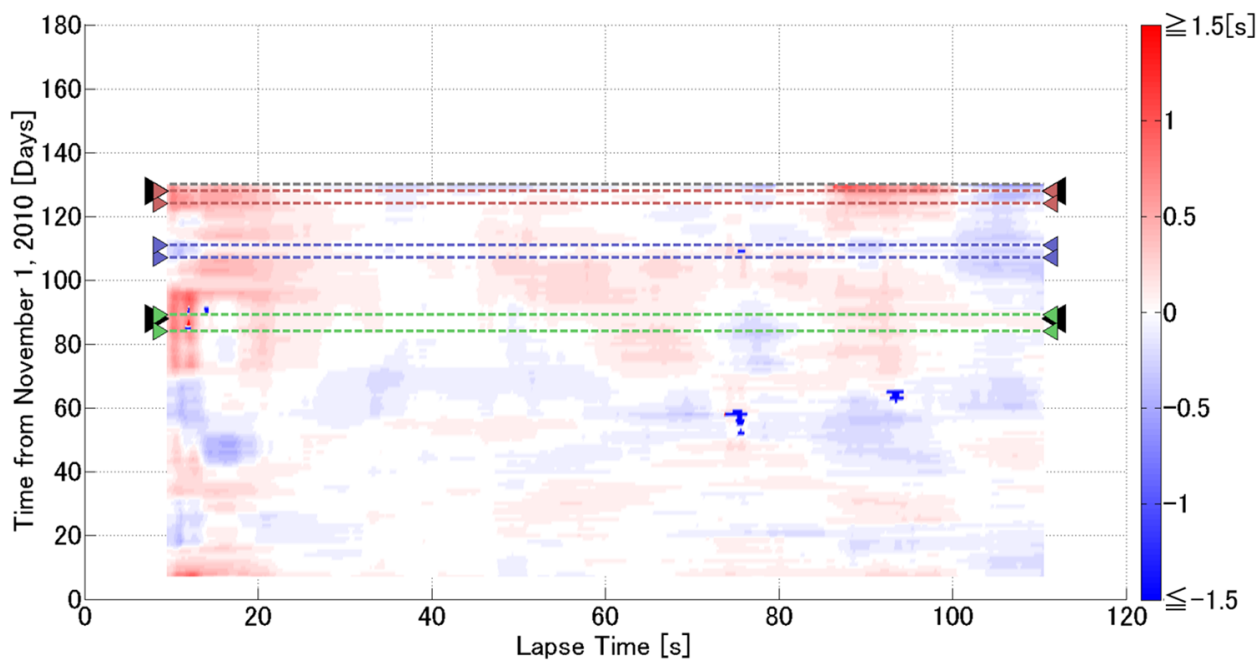

Fig. 4 (See legend on next page.) 
(See figure on previous page.)

Fig. 4 Temporal variations in 15-day ACFs at station S10. a 15-day ACFs calculated from OBS data over 15 continuous days, including the 7 days before and after the given date. The ACFs plotted in red are in the SSE period, and the bottom ACF is the reference ACF, calculated for the month from November 19 to December 19. b The cross-correlation coefficient between the 15-day ACFs and the reference ACF, using a 15-s time window and zero lag time. The period between the black triangles corresponds to the SSE period, and the periods between the red, blue, and green triangles correspond to low-frequency tremor periods in sequences 1, 2, and 3. The magenta broken line represents the main shock. c Phase variation in the 15-day ACFs relative to the reference ACF, using a 15-s time window. Warm colors represent phase progressions, and cold colors represent phase delays. The four pairs of triangles and the magenta broken line correspond to each period and the main shock described in (b)

mainshock, and aftershocks. Because SSEs were detected by GPS data, if another crustal deformation, such as a large earthquake, occurs during an SSE, it is possible that the timing of the end of the SSE is not detected clearly. Moreover, because earthquakes release stress near the hypocenter, an occurrence of large earthquakes ends SSEs that occur near the hypocenter. It is highly likely that the Guerrero SSEs were observed from beginning to end completely because no large earthquakes occurred during the Guerrero SSEs. On the other hand, it is highly likely that the 2011 Tohoku-Oki earthquake occurred before the Tohoku-Oki SSE finished, and it is possible that the Tohoku-Oki SSE was ended suddenly by the 2011 Tohoku-Oki earthquake. If the
2011 Tohoku-Oki earthquake ended the Tohoku-Oki SSE before the velocity recovery period in SSE, it is not surprising that we could not detect velocity recovery in the latter part of the Tohoku-Oki SSE.

\section{Conclusion}

We detected decreases in seismic velocity of no more than $2 \%$ at six stations near the hypocenter of the 2011 Tohoku-Oki earthquake. These decreases were detected after the main shock using seismic interferometry with ambient noise. The largest velocity reduction was observed at S09, located between the hypocenter of the main shock and the slip region of the SSE before the main shock.

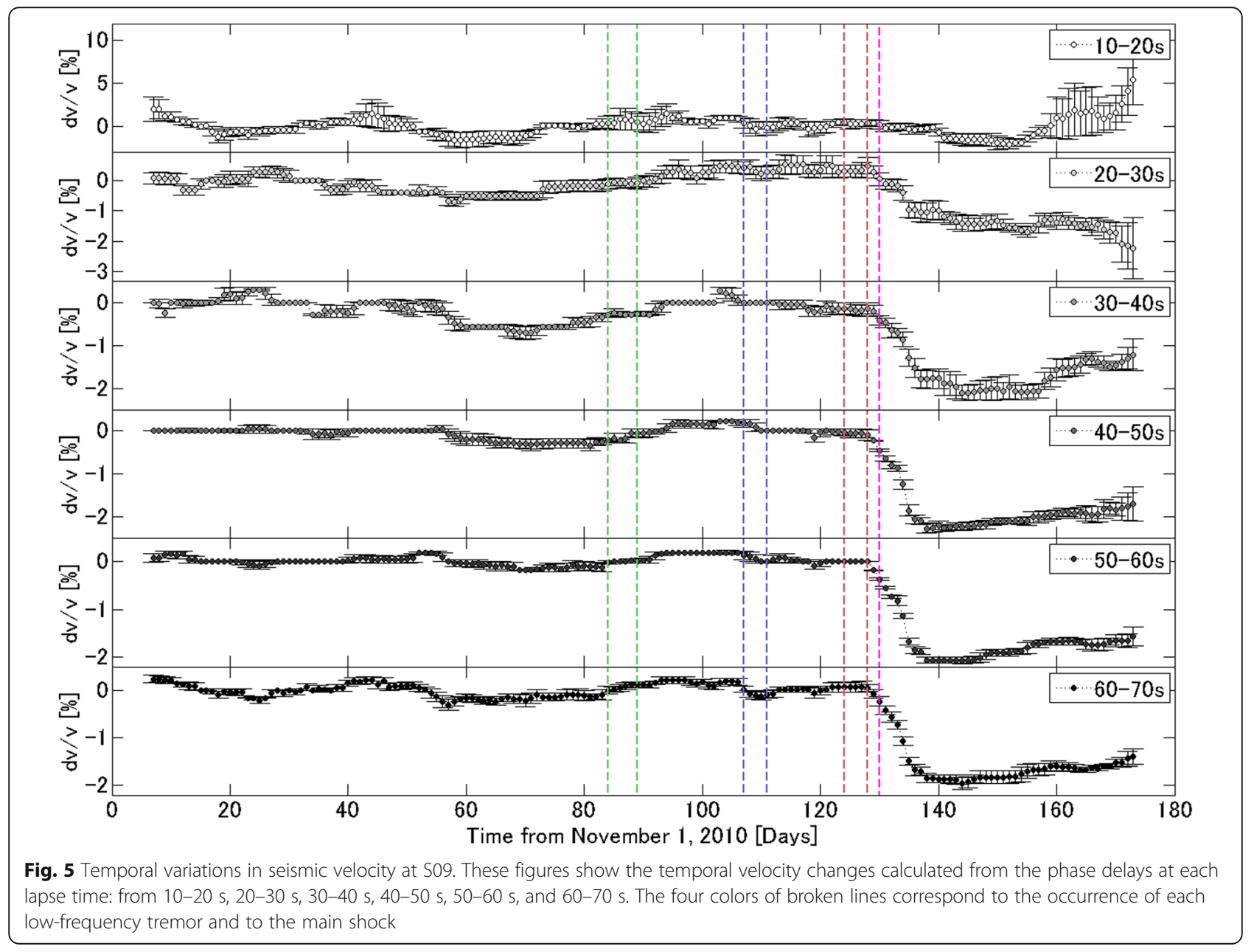


Table 2 Periods used for calculation of variations in crosscorrelation coefficients

\begin{tabular}{ll}
\hline & Period \\
\hline II & November 26, 2010-December 14, 2010 \\
III (sequence 1) & January 9, 2011-January 23, 2011 \\
IV & January 24, 2011-January 29, 2011 \\
V (sequence 2) & February 6, 2011-February 15, 2011 \\
VI & February 16, 2011-February 20, 2011 \\
VII (sequence 3) & February 22, 2011-March 3, 2011 \\
\hline
\end{tabular}

In addition, we detected temporal perturbations in the seismic velocity during the SSE at three stations around the SSE region. The velocities increased a few tenths of a percent immediately after the SSE began and did not change after that. Velocity decreases in the early parts of SSEs and recoveries in the latter parts of SSEs were reported in previous studies; however, we did not detect any velocity recoveries in the latter period.

We observed variations in the 15-day ACFs before a lapse time of $15 \mathrm{~s}$ at most stations installed east of $142.5^{\circ} \mathrm{E}$. These variations were accompanied by low-frequency tremors, although no variations in seismic velocity were detected. Specifically, the 15-day ACFs showed substantial, long-lasting variations during sequence 1 . The variations during sequence 1 included not only the effects of the low-frequency tremors but also the effects of the SSE. All stations were classified based on their characteristics when variations in the 15-day ACFs were observed. Variations in the 15-day ACFs at stations installed between $142.5^{\circ} \mathrm{E}$ and $143^{\circ} \mathrm{E}$ were observed 15 days before sequence 1 , and variations at stations installed east of $143^{\circ} \mathrm{E}$ near the trench were observed during sequence 1 . These regions roughly corresponded to the slip areas of the largest foreshock and SSE. Moreover, the variations in the 15-day ACFs indicated the possibility that low-frequency tremors occurred before sequence 1 between $142.5^{\circ} \mathrm{E}$ and $143^{\circ} \mathrm{E}$.

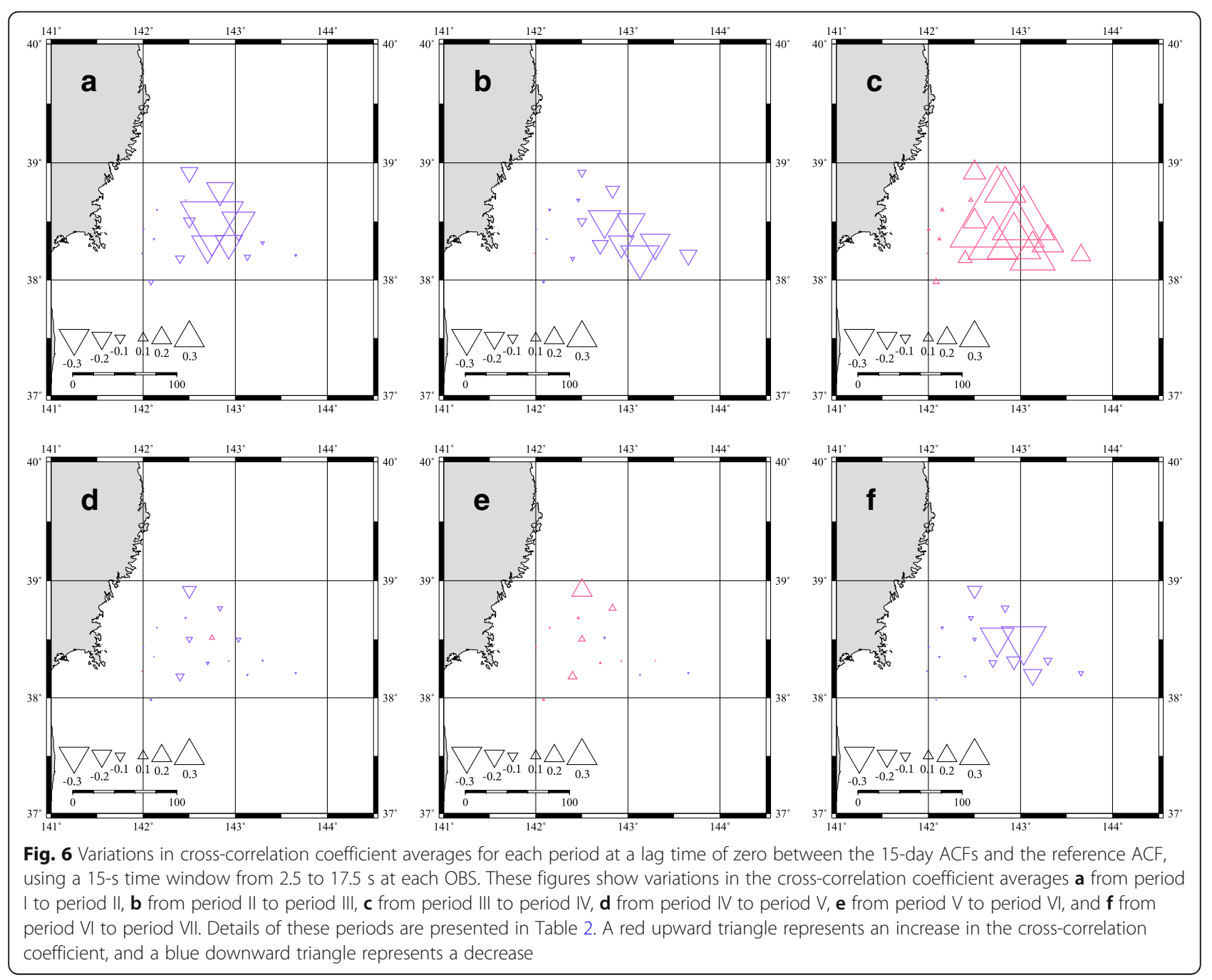




\section{Additional files}

Additional file 1: Temporal variations in 15-day ACFs at Station LS1. (a) 15-day ACFs calculated from OBS data over 15 continuous days, including the 7 days before and after the given date. The ACFs plotted in red are in the SSE period, and the bottom ACF is the reference ACF, calculated for the month from November 19 to December 19. (b) The cross-correlation coefficient between the 15-day ACFs and the reference ACF, using a $15 \mathrm{~s}$ time window and zero lag time. The period between the black triangles corresponds to the SSE period, and the periods between the red, blue, and green triangles correspond to low-frequency tremor periods in Sequences 1, 2, and 3. The magenta broken line represents the main shock (c) Phase variation in the 15-day ACFs relative to the reference ACF, using a $15 \mathrm{~s}$ time window. Warm colors represent phase progressions, and cold colors represent phase delays. The four pairs of triangles and the magenta broken line correspond to each period and the main shock described in (b). (PDF $1740 \mathrm{~kb}$ )

Additional file 2: Temporal variations in seismic velocity at LS1. These figures show the temporal velocity changes calculated from the phase delays at each lapse time: from 10 to 20 s, 20-30 s, 30-40 s, 40-50 s, 50$60 \mathrm{~s}$, and $60-70 \mathrm{~s}$. The four colors of broken lines correspond to the occurrence of each low-frequency tremor and to the main shock. (PDF $51 \mathrm{~kb}$ )

Additional file 3: Temporal variations in 15-day ACFs at Station LS2. (a) 15-day ACFs calculated from OBS data over 15 continuous days, including the 7 days before and after the given date. The ACFs plotted in red are in the SSE period, and the bottom ACF is the reference ACF, calculated for the month from November 19 to December 19. (b) The cross-correlation coefficient between the 15-day ACFs and the reference ACF, using a $15 \mathrm{~s}$ time window and zero lag time. The period between the black triangles corresponds to the SSE period, and the periods between the red, blue, and green triangles correspond to low-frequency tremor periods in Sequences 1, 2, and 3. The magenta broken line represents the main shock. (c) Phase variation in the 15-day ACFs relative to the reference ACF, using a $15 \mathrm{~s}$ time window. Warm colors represent phase progressions, and cold colors represent phase delays. The four pairs of triangles and the magenta broken line correspond to each period and the main shock described in (b). (PDF $1968 \mathrm{~kb}$ )

Additional file 4: Temporal variations in seismic velocity at LS2. These figures show the temporal velocity changes calculated from the phase delays at each lapse time: from 10 to $20 \mathrm{~s}, 20-30 \mathrm{~s}, 30-40 \mathrm{~s}, 40-50 \mathrm{~s}, 50$ $60 \mathrm{~s}$, and $60-70 \mathrm{~s}$. The four colors of broken lines correspond to the occurrence of each low-frequency tremor and to the main shock. (PDF $51 \mathrm{~kb})$

Additional file 5: Temporal variations in 15-day ACFs at Station LS3. (a) 15-day ACFs calculated from OBS data over 15 continuous days, including the 7 days before and after the given date. The ACFs plotted in red are in the SSE period, and the bottom ACF is the reference ACF, calculated for the month from November 19 to December 19. (b) The cross-correlation coefficient between the 15-day ACFs and the reference ACF, using a $15 \mathrm{~s}$ time window and zero lag time. The period between the black triangles corresponds to the SSE period, and the periods between the red, blue, and green triangles correspond to low-frequency tremor periods in Sequences 1, 2, and 3. The magenta broken line represents the main shock. (c) Phase variation in the 15-day ACFs relative to the reference ACF, using a $15 \mathrm{~s}$ time window. Warm colors represent phase progressions, and cold colors represent phase delays. The four pairs of triangles and the magenta broken line correspond to each period and the main shock described in (b). (PDF 1901 kb)

Additional file 6: Temporal variations in seismic velocity at LS3. These figures show the temporal velocity changes calculated from the phase delays at each lapse time: from 10 to 20 s, 20-30 s, 30-40 s, 40-50 s, 50$60 \mathrm{~s}$, and $60-70 \mathrm{~s}$. The four colors of broken lines correspond to the occurrence of each low-frequency tremor and to the main shock. (PDF $51 \mathrm{~kb})$

Additional file 7: Temporal variations in 15-day ACFs at Station LS4. (a) 15-day ACFs calculated from OBS data over 15 continuous days, including the 7 days before and after the given date. The ACFs plotted in red are in the SSE period, and the bottom ACF is the reference ACF, calculated for the month from November 19 to December 19. (b) The cross-correlation coefficient between the 15-day ACFs and the reference ACF, using a $15 \mathrm{~s}$ time window and zero lag time. The period between the black triangles corresponds to the SSE period, and the periods between the red, blue, and green triangles correspond to low-frequency tremor periods in Sequences 1,2 , and 3 . The magenta broken line represents the main shock. (c) Phase variation in the 15-day ACFs relative to the reference ACF, using a $15 \mathrm{~s}$ time window. Warm colors represent phase progressions, and cold colors represent phase delays. The four pairs of triangles and the magenta broken line correspond to each period and the main shock described in (b). (PDF $1839 \mathrm{~kb}$ )

Additional file 8: Temporal variations in seismic velocity at LS4. These figures show the temporal velocity changes calculated from the phase delays at each lapse time: from 10 to 20 s, 20-30 s, 30-40 s, 40-50 s, 50$60 \mathrm{~s}$, and $60-70 \mathrm{~s}$. The four colors of broken lines correspond to the occurrence of each low-frequency tremor and to the main shock. (PDF $51 \mathrm{~kb}$ )

Additional file 9: Temporal variations in 15-day ACFs at Station S01. (a) 15-day ACFs calculated from OBS data over 15 continuous days, including the 7 days before and after the given date. The ACFs plotted in red are in the SSE period, and the bottom ACF is the reference ACF, calculated for the month from November 19 to December 19. (b) The cross-correlation coefficient between the 15-day ACFs and the reference ACF, using a $15 \mathrm{~s}$ time window and zero lag time. The period between the black triangles corresponds to the SSE period, and the periods between the red, blue, and green triangles correspond to low-frequency tremor periods in Sequences 1, 2, and 3. The magenta broken line represents the main shock. (c) Phase variation in the 15-day ACFs relative to the reference ACF, using a $15 \mathrm{~s}$ time window. Warm colors represent phase progressions, and cold colors represent phase delays. The four pairs of triangles and the magenta broken line correspond to each period and the main shock described in (b). (PDF 1922 kb)

Additional file 10: Temporal variations in seismic velocity at S01. These figures show the temporal velocity changes calculated from the phase delays at each lapse time: from 10 to 20 s, 20-30 s, 30-40 s, 40-50 s, 50$60 \mathrm{~s}$, and $60-70 \mathrm{~s}$. The four colors of broken lines correspond to the occurrence of each low-frequency tremor and to the main shock. (PDF $55 \mathrm{~kb})$

Additional file 11: Temporal variations in 15-day ACFs at Station S02. (a) 15-day ACFs calculated from OBS data over 15 continuous days, including the 7 days before and after the given date. The ACFs plotted in red are in the SSE period, and the bottom ACF is the reference ACF, calculated for the month from November 19 to December 19. (b) The crosscorrelation coefficient between the 15-day ACFs and the reference ACF, using a $15 \mathrm{~s}$ time window and zero lag time. The period between the black triangles corresponds to the SSE period, and the periods between the red, blue, and green triangles correspond to low-frequency tremor periods in Sequences 1, 2, and 3. The magenta broken line represents the main shock. (c) Phase variation in the 15-day ACFs relative to the reference ACF, using a $15 \mathrm{~s}$ time window. Warm colors represent phase progressions, and cold colors represent phase delays. The four pairs of triangles and the magenta broken line correspond to each period and the main shock described in (b). (PDF 1943 kb)

Additional file 12: Temporal variations in seismic velocity at S02. These figures show the temporal velocity changes calculated from the phase delays at each lapse time: from 10 to 20 s, 20-30 s, 30-40 s, 40-50 s, 50$60 \mathrm{~s}$, and $60-70 \mathrm{~s}$. The four colors of broken lines correspond to the occurrence of each low-frequency tremor and to the main shock. (PDF $62 \mathrm{~kb}$ )

Additional file 13: Temporal variations in 15-day ACFs at Station S03. (a) 15-day ACFs calculated from OBS data over 15 continuous days, including the 7 days before and after the given date. The ACFs plotted in red are in the SSE period, and the bottom ACF is the reference ACF, calculated for the month from November 19 to December 19. (b) The crosscorrelation coefficient between the 15-day ACFs and the reference ACF, using a $15 \mathrm{~s}$ time window and zero lag time. The period between the black triangles corresponds to the SSE period, and the periods between 
the red, blue, and green triangles correspond to low-frequency tremor periods in Sequences 1, 2, and 3. The magenta broken line represents the main shock. (c) Phase variation in the 15-day ACFs relative to the reference ACF, using a $15 \mathrm{~s}$ time window. Warm colors represent phase progressions, and cold colors represent phase delays. The four pairs of triangles and the magenta broken line correspond to each period and the main shock described in (b). (PDF $1799 \mathrm{~kb}$ )

Additional file 14: Temporal variations in seismic velocity at S03. These figures show the temporal velocity changes calculated from the phase delays at each lapse time: from 10 to $20 \mathrm{~s}, 20-30 \mathrm{~s}, 30-40 \mathrm{~s}, 40-50 \mathrm{~s}, 50-$ $60 \mathrm{~s}$, and $60-70 \mathrm{~s}$. The four colors of broken lines correspond to the occurrence of each low-frequency tremor and to the main shock. (PDF $58 \mathrm{~kb})$

Additional file 15: Temporal variations in 15-day ACFs at Station S04. (a) 15-day ACFs calculated from OBS data over 15 continuous days, including the 7 days before and after the given date. The ACFs plotted in red are in the SSE period, and the bottom ACF is the reference ACF, calculated for the month from November 19 to December 19. (b) The crosscorrelation coefficient between the 15-day ACFs and the reference ACF, using a $15 \mathrm{~s}$ time window and zero lag time. The period between the black triangles corresponds to the SSE period, and the periods between the red, blue, and green triangles correspond to low-frequency tremor periods in Sequences 1,2, and 3. The magenta broken line represents the main shock. (c) Phase variation in the 15-day ACFs relative to the reference ACF, using a $15 \mathrm{~s}$ time window. Warm colors represent phase progressions, and cold colors represent phase delays. The four pairs of triangles and the magenta broken line correspond to each period and the main shock described in (b). (PDF $1960 \mathrm{~kb}$ )

Additional file 16: Temporal variations in seismic velocity at S04. These figures show the temporal velocity changes calculated from the phase delays at each lapse time: from 10 to 20 s, 20-30 s, 30-40 s, 40-50 s, 50$60 \mathrm{~s}$, and $60-70 \mathrm{~s}$. The four colors of broken lines correspond to the occurrence of each low-frequency tremor and to the main shock. (PDF $56 \mathrm{~kb})$

Additional file 17: Temporal variations in seismic velocity at S10. These figures show the temporal velocity changes calculated from the phase delays at each lapse time: from 10 to $20 \mathrm{~s}, 20-30$ s, 30-40 s, 40-50 s, 50$60 \mathrm{~s}$, and $60-70 \mathrm{~s}$. The four colors of broken lines correspond to the occurrence of each low-frequency tremor and to the main shock. (PDF $48 \mathrm{~kb})$

Additional file 18: Temporal variations in 15-day ACFs at Station S14. (a) 15-day ACFs calculated from OBS data over 15 continuous days, including the 7 days before and after the given date. The ACFs plotted in red are in the SSE period, and the bottom ACF is the reference ACF, calculated for the month from November 19 to December 19. (b) The crosscorrelation coefficient between the 15-day ACFs and the reference ACF, using a $15 \mathrm{~s}$ time window and zero lag time. The period between the black triangles corresponds to the SSE period, and the periods between the red, blue, and green triangles correspond to low-frequency tremor periods in Sequences 1, 2, and 3. The magenta broken line represents the main shock. (c) Phase variation in the 15-day ACFs relative to the reference $A C F$, using a $15 \mathrm{~s}$ time window. Warm colors represent phase progressions, and cold colors represent phase delays. The four pairs of triangles and the magenta broken line correspond to each period and the main shock described in (b). (PDF $1933 \mathrm{~kb}$ )

Additional file 19: Temporal variations in seismic velocity at S14. These figures show the temporal velocity changes calculated from the phase delays at each lapse time: from 10 to 20 s, 20-30 s, 30-40 s, 40-50 s, 50$60 \mathrm{~s}$, and $60-70 \mathrm{~s}$. The four colors of broken lines correspond to the occurrence of each low-frequency tremor and to the main shock. (PDF $53 \mathrm{~kb})$

Additional file 20: Temporal variations in 15-day ACFs at Station S15. (a) 15-day ACFs calculated from OBS data over 15 continuous days, including the 7 days before and after the given date. The ACFs plotted in red are in the SSE period, and the bottom ACF is the reference ACF, calculated for the month from November 19 to December 19. (b) The crosscorrelation coefficient between the 15-day ACFs and the reference ACF, using a $15 \mathrm{~s}$ time window and zero lag time. The period between the black triangles corresponds to the SSE period, and the periods between the red, blue, and green triangles correspond to low-frequency tremor periods in Sequences 1,2, and 3. The magenta broken line represents the main shock. (c) Phase variation in the 15-day ACFs relative to the reference $\mathrm{ACF}$, using a $15 \mathrm{~s}$ time window. Warm colors represent phase progressions, and cold colors represent phase delays. The four pairs of triangles and the magenta broken line correspond to each period and the main shock described in (b). (PDF $1911 \mathrm{~kb}$ )

Additional file 21: Temporal variations in seismic velocity at S15. These figures show the temporal velocity changes calculated from the phase delays at each lapse time: from 10 to $20 \mathrm{~s}, 20-30 \mathrm{~s}, 30-40 \mathrm{~s}, 40-50 \mathrm{~s}, 50-$ $60 \mathrm{~s}$, and $60-70 \mathrm{~s}$. The four colors of broken lines correspond to the occurrence of each low-frequency tremor and to the main shock. (PDF $53 \mathrm{~kb})$

Additional file 22: Temporal variations in 15-day ACFs at Station S18. (a) 15-day ACFs calculated from OBS data over 15 continuous days, including the 7 days before and after the given date. The ACFs plotted in red are in the SSE period, and the bottom ACF is the reference ACF, calculated for the month from November 19 to December 19. (b) The crosscorrelation coefficient between the 15-day ACFs and the reference ACF, using a $15 \mathrm{~s}$ time window and zero lag time. The period between the black triangles corresponds to the SSE period, and the periods between the red, blue, and green triangles correspond to low-frequency tremor periods in Sequences 1,2, and 3. The magenta broken line represents the main shock. (c) Phase variation in the 15-day ACFs relative to the reference ACF, using a $15 \mathrm{~s}$ time window. Warm colors represent phase progressions, and cold colors represent phase delays. The four pairs of triangles and the magenta broken line correspond to each period and the main shock described in (b). (PDF $1404 \mathrm{~kb}$ )

Additional file 23: Temporal variations in seismic velocity at S18. These figures show the temporal velocity changes calculated from the phase delays at each lapse time: from 10 to 20 s, 20-30 s, 30-40 s, 40-50 s, 50$60 \mathrm{~s}$, and $60-70 \mathrm{~s}$. The four colors of broken lines correspond to the occurrence of each low-frequency tremor and to the main shock. (PDF $43 \mathrm{~kb})$

Additional file 24: Temporal variations in seismic velocity at S21. These figures show the temporal velocity changes calculated from the phase delays at each lapse time: from 10 to $20 \mathrm{~s}, 20-30 \mathrm{~s}, 30-40 \mathrm{~s}, 40-50 \mathrm{~s}, 50-$ $60 \mathrm{~s}$, and $60-70 \mathrm{~s}$. The four colors of broken lines correspond to the occurrence of each low-frequency tremor and to the main shock. (PDF $53 \mathrm{~kb})$

Additional file 25: Temporal variations in 15-day ACFs at Station S22. (a) 15-day ACFs calculated from OBS data over 15 continuous days, including the 7 days before and after the given date. The ACFs plotted in red are in the SSE period, and the bottom ACF is the reference ACF, calculated for the month from November 19 to December 19. (b) The crosscorrelation coefficient between the 15-day ACFs and the reference ACF, using a $15 \mathrm{~s}$ time window and zero lag time. The period between the black triangles corresponds to the SSE period, and the periods between the red, blue, and green triangles correspond to low-frequency tremor periods in Sequences 1, 2, and 3. The magenta broken line represents the main shock. (c) Phase variation in the 15-day ACFs relative to the reference $\mathrm{ACF}$, using a $15 \mathrm{~s}$ time window. Warm colors represent phase progressions, and cold colors represent phase delays. The four pairs of triangles and the magenta broken line correspond to each period and the main shock described in (b). (PDF $1850 \mathrm{~kb}$ )

Additional file 26: Temporal variations in seismic velocity at S22. These figures show the temporal velocity changes calculated from the phase delays at each lapse time: from 10 to 20 s, 20-30 s, 30-40 s, 40-50 s, 50$60 \mathrm{~s}$, and $60-70 \mathrm{~s}$. The four colors of broken lines correspond to the occurrence of each low-frequency tremor and to the main shock. (PDF $59 \mathrm{~kb})$

Additional file 27: Temporal variations in 15-day ACFs at Station S27. (a) 15-day ACFs calculated from OBS data over 15 continuous days, including the 7 days before and after the given date. The ACFs plotted in red are in the SSE period, and the bottom ACF is the reference ACF, calculated for the month from November 19 to December 19. (b) The crosscorrelation coefficient between the 15-day ACFs and the reference ACF, 
using a $15 \mathrm{~s}$ time window and zero lag time. The period between the black triangles corresponds to the SSE period, and the periods between the red, blue, and green triangles correspond to low-frequency tremor periods in Sequences 1, 2, and 3. The magenta broken line represents the main shock. (c) Phase variation in the 15-day ACFs relative to the reference ACF, using a $15 \mathrm{~s}$ time window. Warm colors represent phase progressions, and cold colors represent phase delays. The four pairs of triangles and the magenta broken line correspond to each period and the main shock described in (b). (PDF $1881 \mathrm{~kb}$ )

Additional file 28: Temporal variations in seismic velocity at S27. These figures show the temporal velocity changes calculated from the phase delays at each lapse time: from 10 to 20 s, 20-30 s, 30-40 s, 40-50 s, 50$60 \mathrm{~s}$, and $60-70 \mathrm{~s}$. The four colors of broken lines correspond to the occurrence of each low-frequency tremor and to the main shock. (PDF $55 \mathrm{~kb})$

Additional file 29: Temporal variations in 15-day ACFs at Station TJT2. (a) 15-day ACFs calculated from OBS data over 15 continuous days, including the 7 days before and after the given date. The ACFs plotted in red are in the SSE period, and the bottom ACF is the reference ACF, calculated for the month from November 19 to December 19. (b) The crosscorrelation coefficient between the 15-day ACFs and the reference ACF, using a $15 \mathrm{~s}$ time window and zero lag time. The period between the black triangles corresponds to the SSE period, and the periods between the red, blue, and green triangles correspond to low-frequency tremor periods in Sequences 1,2, and 3. The magenta broken line represents the main shock. (c) Phase variation in the 15 -day ACFs relative to the reference $\mathrm{ACF}$, using a $15 \mathrm{~s}$ time window. Warm colors represent phase progressions, and cold colors represent phase delays. The four pairs of triangles and the magenta broken line correspond to each period and the main shock described in (b). (PDF $1704 \mathrm{~kb}$ )

Additional file 30: Temporal variations in seismic velocity at TJT2. These figures show the temporal velocity changes calculated from the phase delays at each lapse time: from 10 to 20 s, 20-30 s, 30-40 s, 40-50 s, 50$60 \mathrm{~s}$, and $60-70 \mathrm{~s}$. The four colors of broken lines correspond to the occurrence of each low-frequency tremor and to the main shock. (PDF $44 \mathrm{~kb})$

\section{Abbreviations}

ACF: Autocorrelation Function; CCF: Cross-correlation function; OBS: Ocean bottom seismographs; SSE: Slow slip event

\section{Acknowledgements}

We thank Masatoshi Miyazawa for helpful discussions. We thank Masa Kinoshita, and two anonymous reviewers for their constructive comments. We also thank Editage for the English language revision.

\section{Funding}

This work was supported by the Japan Society for the Promotion of Science (JSPS) KAKENHI grant (\#26257206) and Japan Science and Technology Agency - Japan International Cooperation Agency Science and Technology Research Partnership for Sustainable Development (JST-JICA SATREPS) (\#15543611) grants to M. U. and Y. I., and a JSPS KAKENHI grant (\#26000002) grant to R. H., M. S., Y. I., K. O., and M. U.

\section{Availability of data and materials}

Please contact author for data requests.

\section{Authors' contributions}

MU conceived the study, analyzed the data, and interpreted the analysis results. Yl proposed the topic, collected the data, and helped the interpretation. $\mathrm{KO}$ collected the data and helped the interpretation. $\mathrm{RH}$ and MS collected the data and collaborated with the corresponding author in the construction of manuscript. All authors read and approved the final manuscript.

\section{Authors' information}

M. U. is a student in a doctor's course in division of earth and planetary sciences at Kyoto University. Y. I. is an associate professor at Disaster Prevention Research Institute, Kyoto University. K. O. is an assistant professor at Disaster Prevention Research Institute, Kyoto University. R. H. is a professor at International Research Institute of Disaster Science, Tohoku University. M. $\mathrm{S}$. is a professor at Earthquake Research Institute, The University of Tokyo.

\section{Competing interests}

The authors declare that they have no competing interests.

\section{Publisher's Note}

Springer Nature remains neutral with regard to jurisdictional claims in published maps and institutional affiliations.

\section{Author details}

${ }^{1}$ Kyoto University, Gokasyo, Uji, Kyoto 611-0011, Japan. ${ }^{2}$ Disaster Prevention Research Institute, Kyoto University, Gokasyo, Uji, Kyoto 611-0011, Japan. ${ }^{3}$ Intemational Research Institute of Disaster Science, Tohoku University, 6-6, Aoba, Aramaki, Aoba-ku, Sendai 980-8578, Japan. ${ }^{4}$ Department of Earth and Planetary Science, Tokyo University, 1-1-1, Yayoi, Bunkyo-ku, Tokyo, Japan.

Received: 11 April 2018 Accepted: 13 November 2018

Published online: 19 December 2018

\section{References}

Campillo M, Paul A (2003) Long-range correlations in the diffuse seismic coda. Science 299:547-549

Claerbout JF (1968) Synthesis of a layered medium from its acoustic transmission response. Geophysics 33:264-269

Duvall TL, Jefferies SM, Harvey JW, Pomerantz MA (1993) Time-distance helioseismology. Nature 362:430-432

Hillers G, Campillo M (2016) Fault zone reverberations from cross-correlations of earthquake waveforms and seismic noise. Geophys J Int 204:1503-1517

Hino R, Inazu D, Ohta Y, Ito Y, Suzuki S, linuma T, Osada Y, Kido M, Fujimoto H, Kaneda Y (2013) Was the 2011 Tohoku-Oki earthquake preceded by aseismic Preslip? Examination of seafloor vertical deformation data near the epicenter. Mar Geophys Res. https://doi.org/10.1007/s11001-013-9208-2

Hirose H (2011) Tilt records prior to the 2011 off the Pacific coast of Tohoku earthquake. Earth Planets Space 63:513-518

linuma T, Hino R, Kido M, Inazu D, Osada Y, Ito Y, Ohzono M, Tsushima H, Suzuki S, Fujimoto H, Miura S (2012) Coseismic slip distribution of the 2011 off the Pacific coast of Tohoku earthquake (M9.0) refined by means of seafloor geodetic data. J Geophys Res 117:B07409. https://doi.org/10.1029/ 2012JB009186

Ito $Y$, Hino R (2013) Velocity reduction in an offshore region after the 2011 Tohoku-Oki earthquake, revealed from ocean-bottom seismic records, Proceedings of the 11th SEGJ International Symposium, pp 523-526

Ito $Y$, Hino R, Kido M, Fujimoto H, Osada Y, Inazu D, Ohta Y, linuma T, Ohzono M, Mishina M, Suzuki K, Tsuji T, Ashi J (2013) Episodic slow slip events in the Japan subduction zone before the 2011 Tohoku-Oki earthquake. Tectonophysics 600:14-26

Ito Y, Hino R, Suzuki S, Kaneda Y (2015) Episodic tremor and slip near the Japan trench prior to the 2011 Tohoku-Oki earthquake. Geophys Res Lett 42:1725-1731

Katakami S, Ito Y, Ohta K, Hino R, Suzuki S, Shinohara M (2018) Spatiotemporal variation of tectonic tremor activity before the Tohoku-Oki earthquake. J Geophys Res 123. https://doi.org/10.1029/2018JB016651.

Katakami S, Yamashita Y, Yakihara H, Shimizu H, Ito Y, Ohta K (2017) Tidal response in shallow tectonic tremors. Geophys Res Lett 44:9699-9706

Lockner DA, Byerlee JD, Kuksenko V, Ponomarev A, Sidorin A (1991) Quasi-static fault growth and shear fracture energy in granite. Nature 350(6313):39-42

Minato S, Tsuji T, Ohmi S, Matsuoka T (2012) Monitoring seismic velocity change caused by the 2011 Tohoku-Oki earthquake using ambient noise records. Geophys Res Lett 39(9). https://doi.org/10.1029/2012GL051405

Nimiya H, Ikeda T, Tsuji T (2017) Spatial and temporal seismic velocity changes on Kyushu Island during the 2016 Kumamoto earthquake. Sci Adv 3(11). https://doi.org/10.1126/sciadv.1700813

Nishizawa S, Kanagawa K (2005) Seismic wave velocities in rocks II: velocity anisotropy in metamorphic rocks caused by preferred orientations of minerals and cracks. J Geogr 114(6):949-962

Ohta Y, Hino R, Inazu D, Ohzano M, Ito Y, Mishina M, linuma T, Nakajima J, Osada Y, Suzuki K, Fujimoto H, Tachibana K, Demachi T, Miura S (2012) Geodetic constraints on Afterslip characteristics following the March 9, 2011, Sanrikuoki earthquake, Japan. Geophys Res Lett 39(16). https://doi.org/10.1029/ 2012GL052430 
Poli P, Pedersen HA, Campillo M, the POLENET/LAPNET Working Group (2012) Emergence of body waves from cross-correlation of short period seismic noise. Geophys J Int 188:549-558

Rautian TG, Khalturin VI (1978) The use of coda for determination of the earthquake source Spectrum. Bull Seismol Soc Am 68(4):923-948

Rivet D, Campillo M, Radiguet M, Zigone D, Cruz-Atienza V, Shapiro NM, Kostoglodov V, Cotte N, Cougoulat G, Walpersdorf A, Daub E (2014) Seismic velocity change, strain rate and non-volcanic tremors during the 2009-2010 slow slip event in Guerrero, Mexico. Geophys J Int 196:447-460

Rivet D, Campillo M, Shapiro NM, Cruz-Atienza V, Radiguet M, Cotte N, Kostoglodov V (2011) Seismic evidence of nonlinear crustal deformation during a large slow slip event in Mexico. Geophys Res Lett 38(8). https://doi. org/10.1029/2011GL047151

Sawazaki K, Saito T, Ueno T, Shiomi K (2016) Estimation of seismic velocity changes at different depths associated with the 2014 northern Nagano prefecture earthquake, Japan (Mw6.2) by joint interferometric analysis of NIED hi-net and KiK-net records. Prog Earth Planet Sci 3:36

Snieder R, Grêt A, Douma H, Scales I (2002) Coda wave interferometry for estimating nonlinear behavior in seismic velocity. Science 295:2253-2255

Takagi R, Nakahara H, Kono T, Okada T (2014) Separating body and Rayleigh waves with cross terms of the cross-correlation tensor of ambient noise. J Geophys Res 119(3):2005-2018

Takagi R, Okada T, Nakahara H, Umino N, Hasegawa A (2012) Coseismic velocity change in and around the focal region of the 2008 Iwate-Miyagi Nairiku earthquake. J Geophys Res 117(B6). https://doi.org/10.1029/2012JB009252

Wapenaar K, Draganov D, Snieder R, Campman X, Verdel A (2010) Tutorial on seismic interferometry: part 1-basic principles and applications. Geophysics 75:A195-A209

Wegler U, Nakahara H, Sens-Schönfelder C, Kom M, Shiomi K (2009) Sudden drop of seismic velocity after the 2004 Mw 6.6 mid-Niigata earthquake, Japan, observed with passive image interferometry. J Geophys Res 114(B6). https:// doi.org/10.1029/2008JB005869

Xu ZJ, Song X (2009) Temporal changes of surface wave velocity associated with major Sumatra earthquakes from ambient noise correlation. Proc Natl Acad Sci U S A 106:14207-14212

\section{Submit your manuscript to a SpringerOpen ${ }^{\circ}$ journal and benefit from:}

- Convenient online submission

- Rigorous peer review

- Open access: articles freely available online

- High visibility within the field

- Retaining the copyright to your article

Submit your next manuscript at $\boldsymbol{\nabla}$ springeropen.com 